\title{
Epidemiolgy of spinal cord injury after acute odontoid fractures
}

\author{
James S. Harrop, M.D., Ashwini D. Sharan, M.D., And Gregory J. Przybylski, M.D. \\ Department of Neurosurgery, Thomas Jefferson University Hospital, Philadelphia, Pennsylvania
}

\begin{abstract}
Object. Cervical spinal cord injury (SCI) after odontoid fracture is unusual. To identify predisposing factors, the authors evaluated a consecutive series of patients who sustained SCI from odontoid fractures.

Methods. A consecutive series of 5096 admissions to the Delaware Valley Regional Spinal Cord Injury Center were reviewed, and 126 patients with neurological impairment at the C1-3 levels were identified. Seventeen patients had acute closed odontoid fractures with neurological deficit. Various parameters including demographics, mechanisms of injury, associated injuries, fracture types/displacements, and radiographic cervical canal dimensions were compared between "complete" and "incomplete" spinal cord injured-patients as well as with neurologically intact patients who had suffered odontoid fractures. There were similar demographics, mechanisms of injury, associated injuries, fracture type/displacement, and canal dimensions in patients with complete and incomplete SCIs. However, only patients with complete injury were ventilator dependent. In comparison with patients with intact spinal cords, spinal cord-injured patients were more commonly males $(p=0.011)$ who had sustained higher velocity injuries $(p=0.027)$. The computerized tomography scans of 11 of 17 neurologically impaired patients were compared with those of a random sample of 11 patients with intact spinal cords. Although the anteroposterior diameter $(\mathrm{p}=0.028)$ and cross-sectional area $(\mathrm{p}=$ 0.0004 ) of the cervical spinal canal at the C-2 level were smaller in impaired patients, the displacement of the fragment was not different.

Conclusions. Odontoid fractures are an infrequent cause of SCI. Patients with these injuries typically are males who have smaller spinal canals and have sustained high velocity injuries.
\end{abstract}

\section{KEY WORDS • spinal cord injury • odontoid • cervical spine • tetraplegia}

Cervical spine and SCIs often occur after the patient has sustained traumatic injuries to the head and neck. Although odontoid fractures are the most common axial fracture, associated SCI at this level is less frequent. The relatively larger CSA of the upper cervical spinal canal may reduce the likelihood of neurological injury. The contents of the spinal canal at $\mathrm{C}-1$ include the dens, spinal cord, and CSF space, each comprising approximately one third of the sagittal plane diameter and has been described as Steele's rule of thirds. ${ }^{18}$ With displacement of the odontoid process after fracture, the CSF space can serve to accommodate bone encroachment on the spinal cord without causing injury. The purpose of this study was to examine the epidemiology of SCIs in patients with odontoid fractures.

\section{CLINICAL MATERIAL AND METHODS}

A consecutive series of 5096 admissions to the Delaware Valley Regional Spinal Cord Center during the last

Abbreviations: ADLs = activities of daily living; AP = anteroposterior; ASIA = American Spinal Injury Association; $\mathrm{CSA}=$ cross-sectional area; $\mathrm{CSF}=$ cerebrospinal fluid $; \mathrm{CT}=$ computerized tomography; MVA = motor vehicle accident; $\mathrm{SCI}=$ spinal cord injury. decade was retrospectively reviewed. There were 2749 patients with neurological injuries of which 126 had neurological injuries involving the first three cervical segments, and 17 had closed odontoid fractures without evidence of prior axis injury. In contrast, 209 of the 2347 patients without neurological injuries had odontoid fractures. The medical records and radiographs of patients with odontoid fractures were reviewed. The admission neurological examination was classified according to the ASIA scheme. ${ }^{1}$ The age, sex, mechanism of injury, asso-

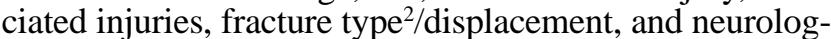
ical examination at follow-up review were determined. Radiographic analysis consisted of evaluation of admission plain radiographs for classification and measurement of displacement. Computerized tomography scanning was performed in only 11 patients with SCIs. The sagittal $\left(D_{s}\right)$ and coronal (D) plane diameters of the spinal canal at the level of the odontoid base were measured inferior to the site of fracture. The sagittal or AP diameter was corrected for gantry angle variability by multiplying the cosine of the gantry angle by the measured AP diameter to obtain the true AP or sagittal diameter. The lateral or coronal diameter of the canal was recorded from measurements of the CT scans obtained at the same axial section as the AP diameter. Assuming a canal shape of a half oval, the CSA was calculated using the formula: $\mathrm{CSA}=\mathrm{p}\left(\mathrm{D}_{\mathrm{s}} / 2 \times \mathrm{D}_{\mathrm{c}} / 2\right) / 2$. 
J. S. Harrop, A. D. Sharan, and G. J. Przybylski

TABLE 1

Summary of characteristics of patients with neurological injury after odontoid fracture*

\begin{tabular}{|c|c|c|c|c|c|c|c|c|c|c|}
\hline $\begin{array}{c}\text { Injury } \\
\text { Level/ASIA } \\
\text { Grade } \dagger\end{array}$ & $\begin{array}{l}\text { Age (yrs), } \\
\text { Sex }\end{array}$ & $\begin{array}{l}\text { Mech- } \\
\text { anism }\end{array}$ & $\begin{array}{l}\text { Type/Dis- } \\
\text { placement } \\
(\mathrm{mm})\end{array}$ & $\begin{array}{c}\mathrm{D}_{\mathrm{S}} \\
(\mathrm{mm})\end{array}$ & $\begin{array}{c}\mathrm{D}_{\mathrm{c}} \\
(\mathrm{mm})\end{array}$ & $\begin{array}{l}\text { CSA } \\
(\mathrm{mm})\end{array}$ & FU & Function & Ventilate & Outcome \\
\hline $\mathrm{C}-2 / \mathrm{A}$ & $86, \mathrm{M}$ & MVA & II,P 8 & 18 & 24 & 170 & na & na & na & dead Day 3 \\
\hline $\mathrm{C}-2 / \mathrm{A}$ & $82, \mathrm{M}$ & fall & II,P 11 & 18 & 27 & 191 & na & na & na & dead Day 4 \\
\hline $\mathrm{C}-3 / \mathrm{A}$ & $89, \mathrm{M}$ & MVA & III none & & & & na & na & na & dead Day 6 \\
\hline $\mathrm{C}-2 / \mathrm{A}$ & $23, \mathrm{M}$ & MVA & III none & & & & na & na & na & dead Day 10 \\
\hline $\mathrm{C}-2 / \mathrm{A}$ & $35, \mathrm{M}$ & fall & II,P 7 & 18 & 22 & 156 & na & na & na & dead Mo 3 \\
\hline $\mathrm{C}-2 / \mathrm{A}$ & $63, \mathrm{~F}$ & fall & II,P 2 & 15 & 23 & 136 & na & na & na & dead Mo 7 \\
\hline $\mathrm{C}-1 / \mathrm{A}$ & $35, \mathrm{M}$ & fall & II none & & & & $\mathrm{C}-2 / \mathrm{A}$ & No & Yes & dead Yr 1 \\
\hline $\mathrm{C}-2 / \mathrm{A}$ & $67, \mathrm{M}$ & dive & II,P 4 & 12 & 29 & 137 & $\mathrm{C}-2 / \mathrm{A} 1 \mathrm{yr}$ & No & Yes & alive Yr 1 \\
\hline $\mathrm{C}-2 / \mathrm{A}$ & $64, \mathrm{M}$ & dive & II,P 3 & 16 & 26 & 163 & C-2/A $1 y r$ & No & Yes & alive Yr 1 \\
\hline $\mathrm{C}-1 / \mathrm{A}$ & $29, \mathrm{M}$ & MVA & II,A 4 & 18 & 22 & 156 & $\mathrm{C}-2 / \mathrm{A} 4 \mathrm{yr}$ & No & Yes & alive Yr 4 \\
\hline $\mathrm{C}-2 / \mathrm{C}$ & $84, \mathrm{M}$ & MVA & II,P 12 & 14 & 25 & 137 & na & No & na & dead Day 2 \\
\hline $\mathrm{C}-2 / \mathrm{C}$ & $83, \mathrm{~F}$ & fall & II,P 13 & 18 & 24 & 170 & na & No & na & dead Day 4 \\
\hline $\mathrm{C}-2 / \mathrm{C}(4)^{\dagger}$ & $42, \mathrm{~F}$ & MVA & III none & & & & C-8/D (87) & Yes & No & alive Yr 3 \\
\hline $\mathrm{C}-2 / \mathrm{B}(0)$ & $46, M$ & other & III none & 25 & 18 & 177 & $\mathrm{C}-3 / \mathrm{C}(22)$ & No & No & alive Yr 9 \\
\hline $\mathrm{C}-2 / \mathrm{C}(15)$ & $33, \mathrm{M}$ & MVA & II,P 7 & 18 & 26 & 184 & C-6/D (56) & No & No & alive Yr 1 \\
\hline $\mathrm{C}-3 / \mathrm{C}(58)$ & $70, \mathrm{M}$ & MVA & II none & & & & C-5/D (97) & Yes & No & alive $\operatorname{Yr} 2$ \\
\hline $\mathrm{C}-3 / \mathrm{D}(85)$ & $37, \mathrm{M}$ & MVA & III none & & & & C-3/D na & Yes & No & alive Mo 3 \\
\hline
\end{tabular}

* $\mathrm{A}=$ anteroposterior; $\mathrm{p}=$ posterior; funcion $=$ independent in ADLs; ventilate $=$ ventilator dependent at follow up.

$\dagger$ Numbers in parentheses are motor scores for incomplete injuries.

These measurements were also obtained from a random sample of neurologically intact patients with odontoid fractures.

Several comparisons of the parameters were made among the groups. First, 10 patients with complete SCIs (ASIA Grade A) were compared with those who had incomplete injuries. Second, 17 spinal cord injured patients (ASIA Grades A-D) were compared with patients who were neurologically intact after odontoid fracture. Finally, CT scans in 11 patients with odontoid fractures and SCIs were compared with 11 control patients without neurological impairment. Statistical analyses were performed using Fisher's test, unpaired t-test, and chi-squared test. Statistical significance was determined at $\mathrm{p}<0.05$.

\section{RESULTS}

The characteristics of the 17 patients with odontoid fractures and neurological injury are summarized in Table 1. Ten patients had complete injuries (ASIA Grade A), typically with a C-2 sensory level. All but two displaced fractures were posteriorly deviated. Only one of seven patients with incomplete injuries had complete motor quadriplegia (ASIA Grade B). Posteriorly displaced fractures were also more common. The age, sex, mechanism of injury, fracture type/displacement, and cervical diameters and CSAs were similar. Seven of 10 patients with complete SCIs died in the 1st year after injury, five of whom died during the first 10 days of hospitalization after ventilatory support was withdrawn. Two of six patients with incomplete injuries died during the first 4 days of hospitalization. Surviving patients with displaced fractures underwent reduction in skeletal traction. All but one patient was immobilized in a halo vest for at least 3 months; another refused halo immobilization and was treated with anterior odontoid screw fixation. Five patients with motor complete injuries survived beyond 5 months; bone union was achieved in three, fibrous union in one, and nonunion in one (further treatment refused). Bone union was achieved in three of four surviving patients with incomplete SCI and adequate follow up, whereas one patient required a subsequent posterior fusion because of nonunion. None of the surviving patients with complete SCIs has experienced neurological improvement, whereas all surviving patients with incomplete SCIs had improved one ASIA grade at 1 year follow-up review. The mean motor score improvement in the patients with incomplete SCI was 46 (range 22-82). ${ }^{1}$ All three patients with motor complete SCIs required ventilatory support at follow up (mean 2.2 years), whereas all patients with incomplete SCIs were successfully weaned from ventilatory support and three regained independence in ADLs (mean 3.9 years).

The 17 neurologically impaired patients were compared with the group having odontoid fractures without neurological injury. Although the mean ages were similar (57 and 65 years, respectively), most patients with neurological injuries were male $(\mathrm{p}=0.011)$; an equal sex distribution was observed in patients with intact spinal cords. Most patients with impairment received high velocity injuries such as those attributable to MVAs ( $p=0.027)$, whereas most patients without impairment experienced falls $(p=0.006)$. Six of the neurologically impaired patients were excluded from the radiographic analysis because adequate admission CT scans were unavailable. Seven of the remaining patients had complete SCIs and four had incomplete SCIs. The mean $\mathrm{D}_{\text {s }}$ measured 17.3 $\mathrm{mm}$ in patients with impaired spinal cords compared with $20.2 \mathrm{~mm}$ in the patients with intact spinal cords $(\mathrm{p}=$ 0.028 ). Similarly, the mean axial CSA at the odontoid base was $162 \mathrm{~mm}^{2}$ (Fig. 1 left) in patients with impaired spinal cords compared with $200 \mathrm{~m}^{2}$ (Fig. 1 right) in those with intact spinal cords $(\mathrm{p}=0.0004)$. There was a mean $25 \%$ reduction in CSA. The difference remained even after excluding the dimensions of the dens. In contrast, the 


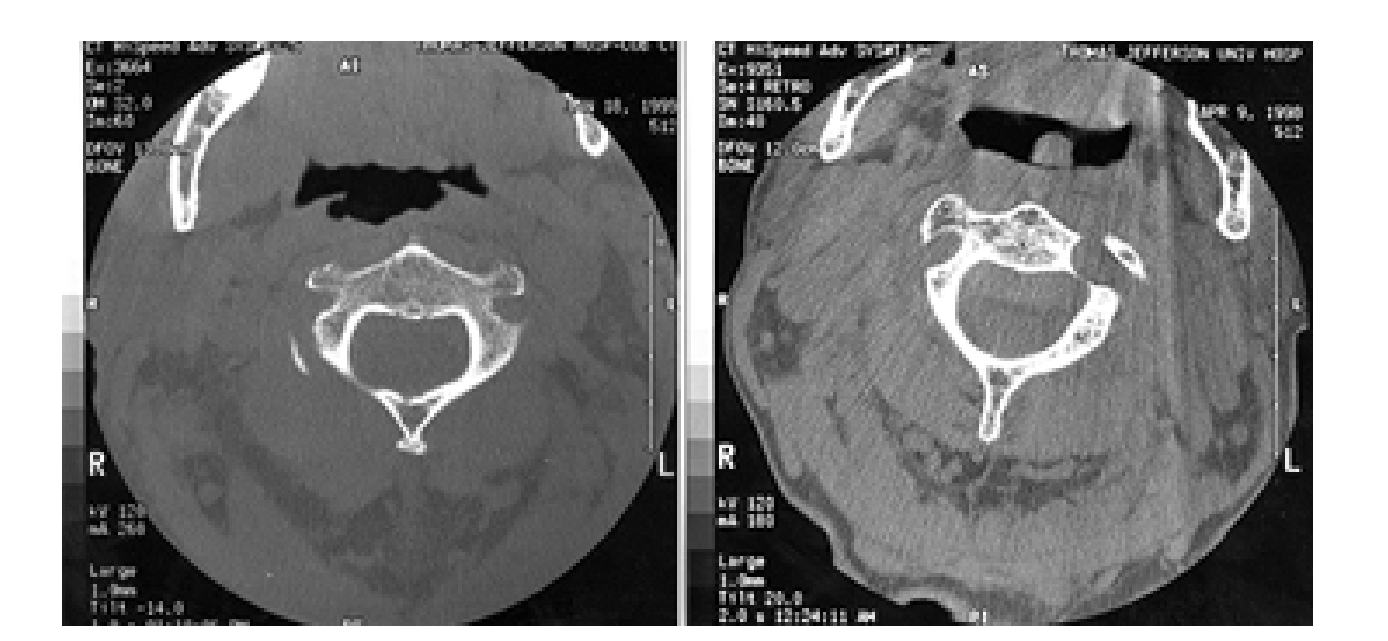

Fig. 1. Left. Axial CT scan obtained at the odontoid base in a 64-year-old man who sustained a C-2 ASIA Grade A SCI from a 3-mm posteriorly displaced Type II odontoid fracture. The $\mathrm{D}_{\mathrm{s}}$ was $16 \mathrm{~mm}$ and the CSA was $163 \mathrm{~mm}^{2}$. Right: Axial CT scan obtained at the odontoid base in an 86-year-old woman without neurological impairment who had an 8-mm posteriorly displaced Type II odontoid fracture. The $\mathrm{D}_{\mathrm{s}}$ was $18 \mathrm{~mm}$ and the CSA was $212 \mathrm{~mm}^{2}$.

$\mathrm{D}_{\text {c }}$ was similar between groups. Moreover, the displacement of the odontoid fragment was not correlated with neurological injury (Fig. 2A and B).

\section{DISCUSSION}

Odontoid fractures are the most common acute traumatic axis fracture. ${ }^{5,8-10}$ The incidence of neurological involvement after odontoid fracture has been reported to be between $0 \%$ and $27 \% .2,3,6,8,10,12$ Others have shown that inadequate treatment of odontoid fractures can result in delayed neurological deterioration and myelopathy secondary to instability. ${ }^{2,7,16}$ However, the factors contributing to acute neurologic injury after odontoid fractures are unknown.

Bohlman ${ }^{4}$ identified 69 of 300 patients with cervical spine injuries as having atlantoaxial trauma. Eleven (15.9\%) had associated SCIs, including five with odontoid fractures; the others had dislocations or penetrating injuries. Hadley and colleagues ${ }^{9,10}$ reported a 5.7\% (13 of 229) frequency, whereas Greene, et al., ${ }^{8}$ reported an $8.5 \%$ (29 of 340) frequency. We observed a similar frequency of $7.5 \%$ (17 of 226 patients). Moreover, a significant correlation between sex and neurological impairment after an odontoid fracture $(\mathrm{p}=0.046)$ was seen. Although the CSA of $177.4 \mathrm{~mm}^{2}$ in men was similar to the CSA of 182.7 $\mathrm{mm}^{2}$ in women, men were more often involved in MVAs.

Neurological injuries in patients with odontoid fractures often occur from high velocity mechanisms such as MVAs. Hadley and colleagues ${ }^{11}$ observed more frequent axis fractures in patients involved in MVAs (68 of 107 patients), particularly if occupants of the vehicle were unrestrained. Few patients with an acute axis fracture who sustain a neurological injury survive to be transported to the hospital. ${ }^{9,17}$ In addition, the National Crash Severity Study showed that $40 \%$ of all cervical SCIs result in immediate death. ${ }^{13}$ These patients may die of asystole at the time of the accident. In fact, 11 of our 17 neurologically impaired patients required cardiopulmonary resuscitation at the scene of the accident. Moreover, persistent cardiac arrhythmias in two patients with motor complete injuries necessitated placement of a permanent pacemaker to treat persistent bradyarrythmia.

The lower incidence of SCI with atlantoaxial injuries is likely due to the larger canal diameter at this spinal level. ${ }^{14,18}$ Reduction in canal diameter in patients developing cervical spondylotic myelopathy was observed in comparison with patients with spondylosis but without myelopathy..$^{15}$ Our measured diameters compared favorably with those reported by others. ${ }^{14} \mathrm{We}$ observed a $25 \%$ reduction in CSA of the canal in patients sustaining neurological injuries compared with patients with intact spinal cords. Moreover, the displacement (direction or distance) of the odontoid was not related to acute neurological injury. Although Hadley, et al. ${ }^{10}$ likewise did not associate displacement with SCI, Dunn and Seljeskog ${ }^{5}$ found more injured patients with posteriorly displaced fractures. Differences in correlating displacement with neurological injury may be related to partial recovery from the original

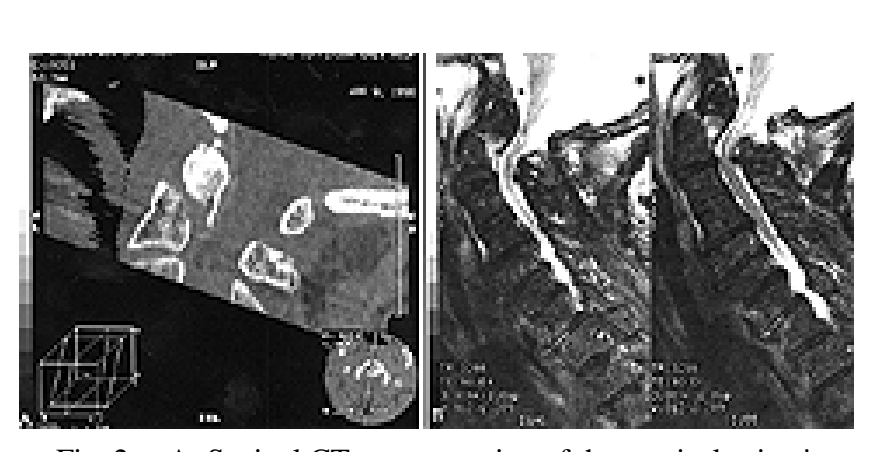

Fig. 2. A: Sagittal CT reconstruction of the cervical spine in a 67-year-old man without neurological impairment who sustained a 12-mm posteriorly displaced odontoid fracture. The $\mathrm{D}_{\mathrm{s}}$ was $20 \mathrm{~mm}$ and the CSA was $212 \mathrm{~mm}^{2}$. B: Sagittal $\mathrm{T}_{2}$ weighted magnetic resonance image demonstrating increased signal intensity at the cervical medullary junction in an 84-year-old man who sustained a C-2 ASIA Grade C SCI from a 12-mm posteriorly displaced Type II odontoid fracture. The $\mathrm{D}_{\mathrm{s}}$ was $14 \mathrm{~mm}$ and the CSA was $137 \mathrm{~mm}^{2}$. 
displacement that occurred during injury and was documented on admission radiographs. The integrity of the tectorial membrane may allow partial or complete reduction.

Finally, patients with motor complete SCIs did not improve, whereas all patients with incomplete SCIs who survived the acute hospitalization period improved, typically by one ASIA grade at 1 year. Motor scores likewise improved. Furthermore, the patients with incomplete SCIs could all be weaned from mechanical ventilation, whereas those with complete SCIs could not. In addition, three of five surviving patients with incomplete SCIs became independent in their ADLs. With better neurological recovery, the patients with incomplete SCIs had a lower 1 -year mortality rate.

\section{CONCLUSIONS}

Acute traumatic odontoid fractures infrequently are associated with neurological injury. The patients sustaining neurological injuries had smaller cervical canal sagittal diameters and CSAs. As a result, these patients have a smaller potential CSF space at the atlantoaxial region in which to accommodate fracture displacement. In addition, high velocity MVAs and male sex correlate with a significant likelihood of sustaining neurological injury. Although the overall mortality rate in patients with neurological injuries after odontoid fractures is high in the 1st year, many patients with incomplete SCIs can regain neurological function and become independent in their ADLs.

\section{References}

1. American Spinal Injury Association: Standards for Neurological Classification of Spinal Injury Patients. Chicago: American Spinal Injury Association, 1996

2. Anderson LD, D'Alonzo RT: Fractures of the odontoid process of the axis. J Bone Joint Surg (Am) 56:1663-1674, 1974

3. Apuzzo MLJ, Heiden JS, Weiss MH, et al: Acute fractures of the odontoid process. An analysis of 45 cases. J Neurosurg 48: 85-91, 1978

4. Bohlman HH: Acute fractures and dislocations of the cervical spine. An analysis of three hundred hospitalized patients and review of the literature. J Bone Joint Surg (Am) 61:1119-1142, 1979
5. Dunn ME, Seljeskog EL: Experience in the management of odontoid process injuries: an analysis of 128 cases. Neurosurgery 18:306-310, 1986

6. Ekong CE, Schwartz ML, Tator $\mathrm{CH}$, et al: Odontoid fracture: management with early mobilization using the halo device. Neurosurgery 9:631-637, 1981

7. Fairholm D, Lee ST, Lui TN: Fractured odontoid: the management of delayed neurologic symptoms. Neurosurgery 38: 38-43, 1996

8. Greene KA, Dickman CA, Marciano FF, et al: Acute axis fractures. Analysis of management and outcome in 340 consecutive cases. Spine 22:1843-1852, 1997

9. Hadley MN, Browner C, Sonntag VKH: Axis fractures: a comprehensive review of management and treatment in 107 cases. Neurosurgery 17:281-290, 1985

10. Hadley MN, Dickman CA, Browner CM, et al: Acute axis fractures: a review of 229 cases. J Neurosurg 71:642-647, 1989

11. Hadley MN, Sonntag VK, Grahm TW, et al: Axis fractures resulting from motor vehicle accidents: the need for occupant restraints. Spine 11:861-864, 1986

12. Hanigan WC, Powell FC, Elwood PW, et al: Odontoid fractures in elderly patients. J Neurosurg 78:32-35, 1993

13. Huelke DF, O'Day J, Mendelsohn RA: Cervical injuries suffered in automobile crashes. J Neurosurg 54:316-322, 1981

14. Lang J: Clinical Anatomy of the Cervical Spine. Wallace SA, translator. New York: Thieme, 1993, pp 79-114

15. Payne E, Spillane JD: The cervical spine. An anatomic-pathological study of 70 specimens (using a special technique) with particular reference to the problem of spondylosis. Brain 80: $571-596,1957$

16. Osgood RB, Lund CC: Fractures of the odontoid process. $\mathbf{N}$ Engl J Med 198:61-72, 1928

17. Schneider RC, Livingston KE, Cave AJE, et al: "Hangman's fractures" of the cervical spine. J Neurosurg 22:141-154, 1965

18. Spence KF Jr, Decker S, Sell KW: Bursting atlantal fracture associated with rupture of the transverse ligament. J Bone Joint Surg (Am) 52:543-549, 1970

Manuscript received April 25, 2000.

Accepted in final form May 23, 2000.

This work was supported in part by the Regional Spinal Cord Injury Center of Delaware Valley Model SCI System Grant No. H133N00027 to Thomas Jefferson University Hospital from the National Institute for Disability Research and Rehabilitation.

Address reprint requests to: James Harrop, M.D., 1015 Chestnut Street, Suite 1400, Philadelphia, Pennsylvania 19107. email: EJHarrop@aol.com 